\title{
Electroencephalography at the time of Covid-19 pandemic in Italy
}

\author{
Giovanni Assenza ${ }^{1}$ (1) Jacopo Lanzone ${ }^{1} \cdot$ Lorenzo Ricci $^{1} \cdot$ Marilisa Boscarino $^{1} \cdot$ Mario Tombini $^{1}$. \\ Carlo Andrea Galimberti ${ }^{2} \cdot$ Lara Alvisi $^{3} \cdot$ Laura Tassi $^{4} \cdot$ Lidia Broglia $^{5} \cdot$ Vincenzo Di Lazzaro $^{1} \cdot$ Oriano Mecarelli $^{6}$
}

Received: 1 June 2020 / Accepted: 21 June 2020 / Published online: 26 June 2020

(C) Fondazione Società Italiana di Neurologia 2020

\begin{abstract}
Objective During the Covid-19 pandemic, government restrictions limited health care to urgent needs. Neurophysiology centers had to suddenly reschedule their activities, with a lack of specific recommendations about electroencephalography (EEG) execution. During the pandemic phase 1, we launched an online survey to understand the flaws and strengths of the EEG management in Italy at the time of Covid-19 pandemic.

Methods A 45-item online survey (published from April 16 to 30, 2020), endorsed by the Italian Society of Clinical Neurophysiology (SINC), the Italian League Against epilepsy (LICE), and the Italian Association of Neurophysiology technologists (AITN), collected EEG management data (EEG's number and type, indications, personnel and patients safety, devices' sanification) during the Covid-19 pandemic.

Results We received responses from 206 centers. The number of EEGs performed was reduced by $76 \pm 20 \%$, and several types of specific EEG (video-EEG, ambulatory-EEG, LTM, polysomnography) were reduced at a minimum. Half of the centers performed inpatient EEGs only for urgencies. Repetitive seizures, encephalitis, and non-convulsive status epilepticus were the most common indications. Covid-19-positive patients received less EEG than negative ones $(p<0.0001)$. EEG requests came mainly not only from neurologists $(n=176)$ but also from general practitioners $(n=40)$, emergentists $(n=79)$, intensivists $(n=72)$, and other specialists $(n=53)$. Those centers which continued performing outpatient EEG examinations were instructed to perform the EEG after a Covid-19-related symptom screening for patients and using personal protective equipment (PPE) through all the procedure. Inpatient EEGs were performed using FFP2/FFP3 masks by neurophysiology technologists in only $50 \%$ of cases. Patients executed hyperventilation only for real clinical needs, but often (56\%) with a mask.

Conclusions Italian neurophysiology centers strongly adhered to government restrictions of lockdown. Some issues emerged, ranging from the evaluation of a proper indication for EEG, technical procedures of EEG recording, and protection of neurophysiology technicians.
\end{abstract}

Keywords EEG $\cdot$ Covid-19 $\cdot$ Sars-Cov-2 virus $\cdot$ Neurophysiology $\cdot$ Italy

\section{Introduction}

Italy is facing an unprecedented health emergency represented by the Covid-19 pandemic due to SARS-CoV-

Giovanni Assenza

g.assenza@unicampus.it

1 Unit of Neurology, Neurophysiology, Neurobiology, Department of Medicine, University Campus Bio-Medico of Rome, Via Álvaro del Portillo, 200, 00128 Rome, Italy

2 IRCCS Mondino Foundation, Pavia, Italy
2 virus. The government impositions to limit the spreading of infection included heavy restrictions of health care services throughout the countries hit by SARSCoV-2 virus.

3 IRCCS Institute of Neurological Sciences of Bologna, Department of Biomedical and Neuromotor Sciences, University of Bologna, Bologna, Italy

4 “Claudio Munari” Epilepsy Surgery Center, ASST Niguarda Hospital, Milan, Italy

5 UOC Neurology, San Filippo Neri Hospital, ASL Roma 1, Rome, Italy

6 Department of Human Neurosciencies, Sapienza University of Rome, Rome, Italy 
The Italian Ministry of Health, the Centers for Disease Control and Prevention, and the Centers for Health Care and Medical Assistance Services recommended the suspension of all elective and "non-urgent" procedures, including those concerning radio-diagnostic examinations, surgery, and neurophysiological exams [1-3]. Routine outpatient visits were suspended, postponed, and replaced, when possible, by remote assistance (telephone, e-mail, or video-audio-conference consultations) [4-6]. However, several issues about the execution of health care assistance during the lockdown period remained unanswered. This is the case for the so-called elective or nonurgent procedures, being highly variable depending on the clinical setting (outpatient and inpatient examinations), type of procedure, and the status of the patient according to the SARS$\mathrm{CoV}-2$ virus infection. Furthermore, the Italian Government did not provide any specific instructions concerning the use of personal protective equipment (PPE) for different procedures and the modalities of protection and disinfection of medical devices. However, despite the Covid-19 pandemic and its inherent risks, urgent medical assistance is often required for patients with neurological conditions, in order to avoid harmful delays in diagnosis and treatments and the related negative consequences on both patients and the health care system.

The decision to perform some diagnostic procedures could be particularly challenging for some neurophysiological examinations, such as the electroencephalogram (EEG). Actually, even if the EEG recording does not represent an emergency in most cases, it may not be indefinitely delayed for some acute/subacute neurological conditions.

The sudden and rapid spreading of SARS-CoV-2 virus infection in Italy and in other countries out of China did not allow to produce prompt recommendations about the EEG execution during the first weeks of Covid-19 pandemic. Thus, Italian centers had to customize their EEG activities on local basis, waiting for international and national recommendation from scientific societies.

Impending the pandemic phase 1, we published an online survey endorsed by the three most representative Italian scientific neurophysiological societies, (i) the Italian Society of Clinical Neurophysiology (SINC), (ii) the Italian League Against Epilepsy (LICE), and (iii) the Italian Association of Neurophysiology Technologists (AITN), to obtain information on the different strategies adopted by Italian neurophysiological centers in facing with EEG execution during the Covid-19 pandemic, in order to detect the flaws and strengths for possible future programming of neurophysiological activities in emergency conditions.

\section{Material and methods}

A 45-item online survey was created using the free open access Google ${ }^{\mathrm{TM}}$ Forms (https://www.google.com/forms/ about/). The survey included an informed consent, making possible for those who did not agree with its terms of use, to end the survey without further questions. No personally identifiable information was collected, and data were treated according to the European regulation, GDPR no. 2016/679. The survey was e-mailed to the members on April 16, 2020 on the websites of the SINC, LICE, and AITN societies. It was closed on 11:59 p.m. April 30, 2020.

The questionnaire contained the following sections:

- Introduction with a brief description of the aim of the study

- Informed consent (mandatory)

- Center denomination and location

- Professional status (physician/technician and specialty)

- Monthly number of EEG in pre-Covid-19 period (before lockdown restrictions, i.e., March 11, 2020) and in the Covid-19 period (from March 11, 2020)

- Availability to perform EEG according to patients' age (newborns, children, adults)

- Type of EEG performed in the pre-Covid-19 period and in the Covid-19 period (standard EEG, ambulatory EEG, video-EEG, long-term monitoring (LTM), polysomnography) - Changes in EEG recording according to urgency (routine or urgent) and clinical settings (inpatients or outpatients)

- Hyperventilation use and modalities

- Anamnestic data, biometric data a use of PPE by Covid19-negative inpatients and outpatients for EEG recording - Adopted procedures for managing accompanying persons

- PPE used by technicians for EEG recording in Covid-19negative and positive inpatients and outpatients

- Type of electrodes and EEG cup used for Covid-19negative and Covid-19-positive inpatients and outpatients

- Disinfection of non-disposable devices

- Availability of Covid-19-dedicated EEG devices

- Number of SARS-CoV-2 virus infections of hospital personnel

- Availability and use of a Covid-19-dedicated psychological assistance

Differences among EEG recordings between the pre-Covid19 and the Covid-19 period and between Covid-19-positive and Covid-19-negative patients were compared by $\chi^{2}$ test.

Significancy was set at $p$ value $<0.05$. Data are shown as mean \pm standard deviation or median and ranges as appropriate.

\section{Results}

\section{Participants}

We received 216 different responses to the questionnaire. Ten responses were not furtherly analyzed being duplicate from 
the same neurophysiology center. Thus, we considered 206 answers: 103 answers came from physicians and 98 from technicians, and 5 did not answer about their job status for privacy concerns.

Among physicians, 70 were neurologists, 19 child neurologists, 10 neurophysiologists, 3 pediatricians, and 1 neurosurgeon.

One hundred two centers were located in the North $(49 \%$; Piemonte 31, Lombardia 32, Emilia-Romagna 17, Liguria 5, Trentino Alto Adige 2, Friuli Venezia Giulia 3, Veneto 12), 49 in the Centrum (24\%; Toscana 13, Umbria 5, Lazio 22, Marche 6, Molise 1, Abruzzo 2), and 55 in the South and islands (27\%, Basilicata 1, Calabria 6, Campania 12, Puglia 18, Sardegna 7, Sicilia 11) of Italy.

\section{EEG activity}

The number of weekly EEG performed in the pre-Covid-19 period was $46.1 \pm 32.5$, while in the Covid-19 period, it was reduced to $11 \pm 12$ (a reduction by $76 \pm 20 \%$ SD; Fig. 1). Seven of centers did not record EEG at all in the Covid-19 period.

In the pre-Covid-19 period, 81 centers usually performed EEG in adults, children, and newborns, 48 centers performed EEG only in adults, 46 in adults and children, 18 only in children, and 5 in children and newborns.

In the pre-Covid-19 period, standard EEG was recorded in 193 centers, video-EEG in 137, polysomnography in 86, ambulatory EEG in 65, and long-term EEG for pre-surgical evaluation in 52.

During the Covid-19 period, standard EEG was recorded in 184 centers (5\% reduction), video-EEG in 93 (32\% reduction), polysomnography in 40 (53\% reduction), ambulatory EEG in 14 (78\% reduction), and long-term EEG for presurgical evaluation in 10 (81\% reduction; Fig. 2).

During the Covid-19 period, EEG was recorded for urgent inpatients in 109 centers, for routine and urgent inpatients in

\section{EEG CHANGES DURING LOCKDOWN} (N/WEEK)

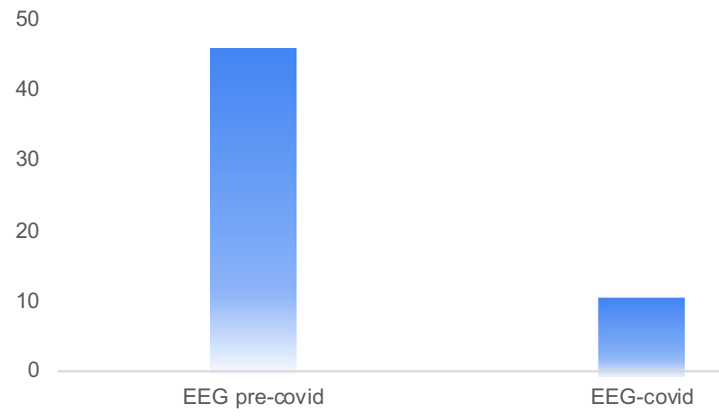

Fig. 1 EEG changes during the Covid-19 pandemic. Pre-Covid-19 period: before governmental restrictions (March 11, 2020). Cov-19 period: after governmental restrictions (from March 11, 2020 to the end of the present questionnaire publication, April 30, 2020)
73 , for urgent outpatients in 106, and for routine and urgent outpatients in 8 . No changes in respect to usual activity were declared in 4 centers, while no EEGs at all were performed in 9 (Fig. 3).

Hyperventilation was discontinued as standard EEG provocation technique in 25 centers, 42 performed it only when there was a strong clinical indication, 121 performed it with mask, and 15 performed it without mask.

A total of 202 participants declared that EEG laboratory activity changed during the pandemic period. In particular, there was a reduction in activity in 200 centers, while an increase was reported in 2 centers (one center from Como and 1 center from Brescia, both of them located in Lombardia, the Italian region with the highest number of SARS-CoV-2 virus infections; www.salute.gov.it).

A total of 44 centers reported a change in the duration of EEG during the pandemic period with shortening in 35 centers and an increase in 9.

\section{Inpatients}

During the recording of inpatients' EEG, 164 centers required the patient wearing a mask, 105 verified Sars-Cov-2 infection's symptoms, 20 performed EEG only with a negative nasopharyngeal swab for SARS-Cov2 virus, no precautions were reported in 7 centers, and no EEGs were performed in 12 centers.

In Covid-19-positive inpatients, EEG was performed in case of repetitive seizures in 158 centers, for suspected encephalitis in 132, for non-convulsive status epilepticus (NCSE) in 118, for brain death evaluation in 87, for prognostic assessment after post-anoxic coma in 86 , after a first seizure in 81 , for neonatal seizures in 64 , and for infantile spasms in 45.

In Covid-19-negative inpatients, EEG was performed in case of repetitive seizures in 189 centers, for suspected encephalitis in 161, for NCSE in 137 centers, for prognostic assessment after post-anoxic coma in 126, for brain death evaluation in 121 , after a first seizure in 113 , for neonatal seizures in 96, and for infantile spasms in 72 .

Covid-19-positive patients received fewer EEG examinations compared with Covid-19-negative patients $\left(\chi^{2}\right.$, $p<0.0001$ ). In Covid-19-positive inpatients, EEGs were performed in 76 centers, for a total of 1010 EEGs in the Covid-19 period (35-50 days), with a mean weakly number of 265 EEG requests (229 during ordinary work time, 27 on-call).

\section{Outpatients}

In outpatient's EEG, all centers required some precautions. A total of 168 centers required patients wearing a mask, 141 verified Covid-19 symptoms, 20 performed EEG only with a negative nasopharyngeal swab for Covid-19, and 4 measured body temperature. 
Fig. 2 EEG type changes during the Covid-19 pandemic. LTM long-term monitoring videoEEG, PSG polysomnography, StEEG standard EEG

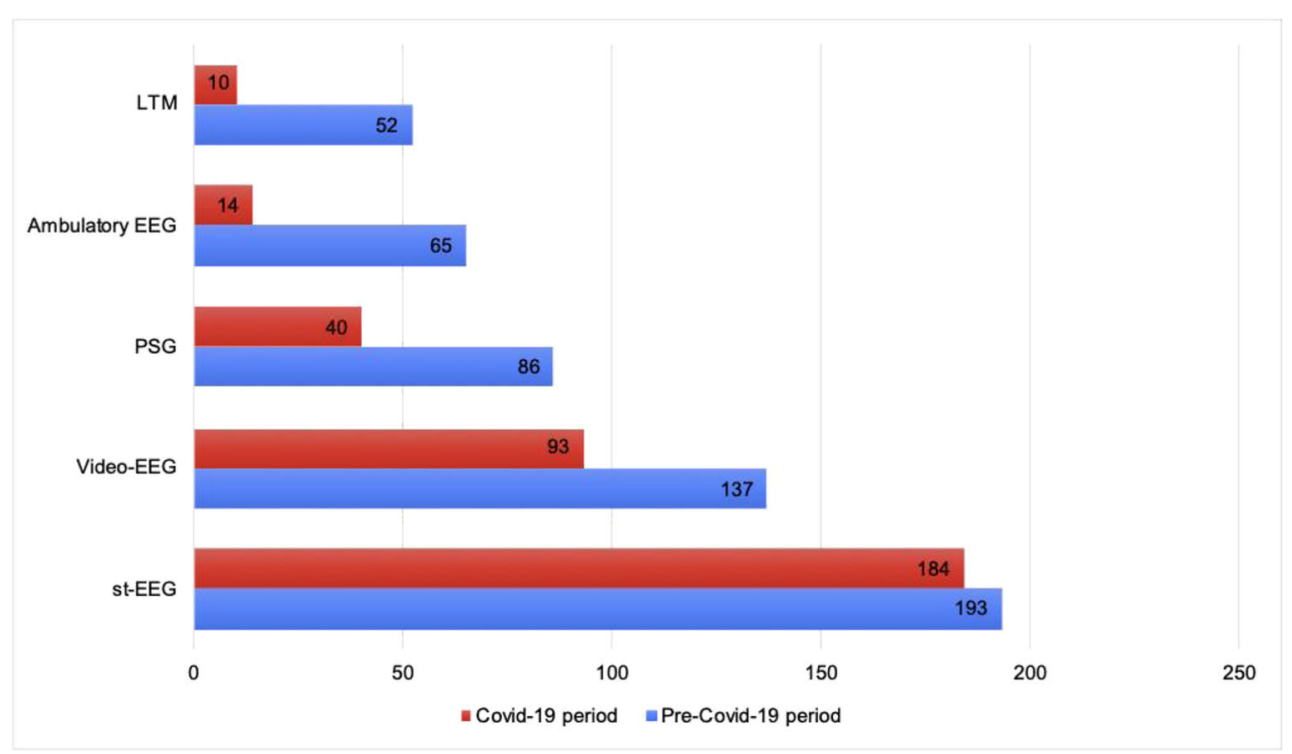

In 121 centers, patients had to stay alone in the EEG laboratory; in 73 and respectively 47 centers, an accompanying person was allowed to enter in the lab for children or patients with intellectual disability. The accompanying people had to wear a mask in 94 centers, mask and gloves in 105, and none of them in 5 .

The EEG request (for both inpatients and outpatients) could be performed by neurologist/neurosurgeon in 176 centers, general practitioners in 40 centers, emergentists in 79 , intensivists in 72 , and by any specialist in 53 centers.

\section{NPT safety and technical device management}

During outpatients' EEG recording, neurophysiology technicians (NPT) wore a surgical mask in 184 centers, FFP2 mask in 56 centers, and FFP3 in 10 centers.
During inpatients' EEG recording, NPT wore a surgical mask in 166 centers, FFP2 mask in 89 centers, and FFP3 in 20 centers. NPT of all centers wore gloves in every kind of EEG.

During the pandemic period, NPT used cup electrodes in 70 centers, bridge electrodes in 86 , subdermal needles in 84 , and pre-wired cap in 116.

In pre-Covid-19 period, non-disposable cups and electrodes were disinfected in 124 centers, while during the Covid-19 period, disinfection was performed in all centers.

In Covid-19-positive patients, non-disposable material was used in 59 centers and disinfection of non-disposable material was performed after each test according to the institutional indications.

Fifty-nine centers with a Covid-19-specific ward used a dedicated EEG machine (portable EEG machine in 55, fixed EEG machine in 4); 107 centers used only antiseptic solutions
Fig. 3 EEG clinical indications and settings

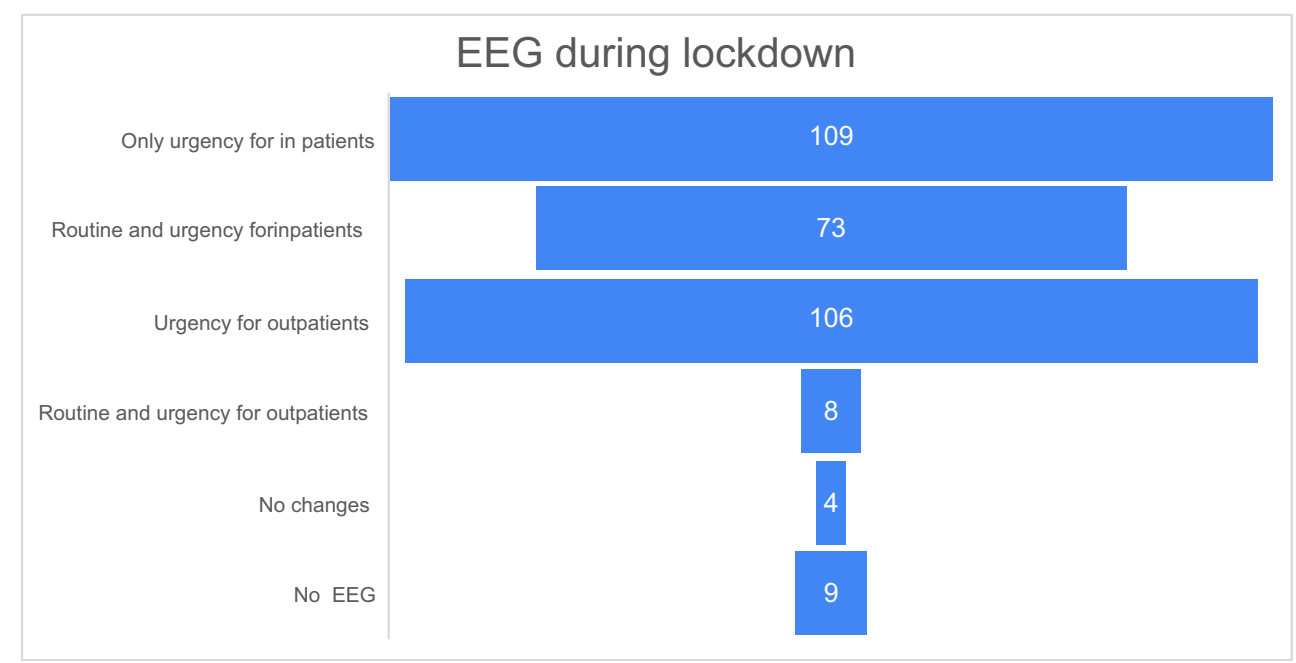


to disinfect the machine while 42 centers also applied a physical protection on EEG machine.

A SARS-CoV-2 virus contagion of hospital employees was reported in 106 centers for a total of 1564 persons (doctors, technicians, nurses, administrative, and social-health care workers).

In 119 centers, a Covid-19-dedicated psychological counseling support service was available, but only 3 centers declared to have used it.

\section{Discussion}

Our survey provided a glimpse of the working activities of the Italian neurophysiological centers during the Covid-19 pandemic. Participation to the present survey was highly representative if we consider that Italian SINC-endorsed centers are 106 and LICE-endorsed centers are 72. This could mean that almost the totality of endorsed (and thus certified) Italian neurophysiological centers participated and several other centers filled the questionnaire. Topographical distribution of the centers also reflected those reported in the web site of the two scientific societies, with most of the centers located in Northern Italy, less in the Centrum and the South (including islands) of the Country. Geography of the centers made even more reliable our estimate of the Covid-19 pandemic impact on neurophysiology activity because the Northern regions received the most of the contagions in Italy (data available on www.salute.gov.it). Actually, our participants reported more than one thousand of Sars-Cov-2 virus contagions among the hospital employees.

The reported reduction of more than $70 \%$ of EEG activity confirmed the good adherence of the centers to the governmental restrictions. Moreover, the type of EEG decrease reflects the restriction of EEG indications only for urgent clinical conditions. While almost all centers recorded regularly standard EEGs, a minor reduction was observed for videoEEGs, but a deep decline in pre-surgical long-term monitoring studies, ambulatory EEGs, and polysomnographic studies was observed. About half of the centers performed EEG in urgent cases for both inpatients and outpatients, while ensuring routine EEG for inpatients. Only a minority of the centers kept on executing routine outpatient EEG and did not experience any modifications in their usual activities.

Participating centers were discordant on the execution of hyperventilation, which was not performed in 25 centers, performed only for real clinical needs in 42 , without mask in 15 , with mask in 121 centers. This observation is surprising since hyperventilation is an activating procedure, which should not be executed with a mask. Even if data on putative pathophysiological pathways inducing epileptiform EEG "activation" during hyperventilation is not conclusive [7], the main hypothesis point towards hemodynamic cerebral changes.
Hyperventilation decreases arterial $\mathrm{pCO}_{2}$ and leads to vasoconstriction of precapillary arterioles which, as a consequence, reduces cerebral blood flow [8]. Thus, wearing a mask produces the opposite effect because it increases the $\mathrm{pCO}_{2}$, thus resulting in cerebral vasodilatation. For these reasons, hyperventilation should never be executed with mask and should be reserved, in this pandemic period, only for strong clinical indications. This approach from the Italian centers was potentially encouraged by a lack of specific recommendations by scientific societies, especially during the early weeks of the pandemic.

The duration of standard EEG examination remained unchanged in most centers, while few centers reduced it and a minority declared an increase of mean EEG duration during the pandemic.

When analyzing the clinical indications for inpatient EEG, repetitive seizures, suspicion of encephalitis, NCSE, and brain death evaluation were the most frequent, followed by diagnostic assessment after a first seizure, neonatal seizures, and infantile spasms.

These indications were valid for both Covid-19-positive and Covid-19-negative patients, even if Covid-19-positive patients received fewer EEGs than the Covid-19-negative ones. The total number of EEGs executed in Covid-19-positive patients was more than one thousand.

In both inpatients and outpatients, the mask for the patient and a negative anamnesis for SArs-Cov-2 virus presenting symptoms were required during EEG examination in most centers, while only about $10 \%$ of centers required a negative Sars-Cov2 swab.

Half of the centers allowed an accompanying person to enter during patients' EEG recordings, especially for pediatric or disabled patients, but only after wearing mask and gloves.

Unfortunately, the indication for EEG was provided not only by neurologists but also by emergentists, intensivists, general practitioners and other specialists. In accordance with International [9] and the only recently published Italian recommendations (www.lice.it; www.sinc-italia.it; www.aitn.it; not available at the moment of the present questionnaire publication) [10], we think that this approach to EEG's prescription executed by a non-neurologist is not a good clinical practice considering the need of reducing the total number of the EEG and of limiting its recording to the only urgent cases. Actually, since the beginning of the emergency period, international [1] and Italian governmental agencies [11] recommended the suspension of all elective and "non-urgent" procedures, including radiological tests, surgery, and neurophysiological exams. EEG is a neurophysiological technique that could be extremely useful in case of acute/subacute onset of neurological symptoms for diagnostic, therapeutic, and prognostic assessments. However, these clinical conditions may represent diagnostic challenges also for the neurologists (e.g., non-convulsive status epilepticus, encephalitis, coma). 
Therefore, giving the possibility to prescribe EEG to other specialists without a neurological clinical background could result in an increasing number of unnecessary or avoidable EEGs. For these reasons, the abovementioned recommendations suggest involving the neurologist in the evaluation of the EEG's request, in particular in those scenarios of pandemic lockdown where personnel restriction is present.

NPT were always protected by mask and gloves, but only half of them could wear an appropriate (FFP2 or FFP3) mask for the execution of inpatient EEGs. In about 4 out of 5 centers with a Covid-19-specific ward, EEG was recorded with a dedicated device. When describing EEG machine disinfection and precautions, respondents reported in only one-third of the cases to use a physical protection (plastic film or cover) in addition to the standard disinfectant solutions. During the Covid-19 pandemic, non-disposable electrodes were disinfected in all centers, but it is worth mentioning that only half of them sanitized them before the pandemic after each test. However, there are not Italian guidelines on the technical requirements for EEG execution, and the recommendation of American Clinical Society of Neurophysiology state that "Electrodes must be disinfected with appropriate procedures and transmissionbased precautions taken after recording from patients with contagious diseases" [12]. Thus, there is not a clear indication to sanitize the EEG electrodes after every single use regardless of the infectious status of the patient. In our opinion, in the present scenario, this represents a serious gap in international recommendations for EEG technical execution.

\section{Conclusions}

Italian neurophysiological centers strongly adhered to the government restrictions adopted during the Covid-19 lockdown. The strategies of Italian centers were absolutely congruous with real clinical needs of the phase 1 pandemic scenario. However, some flaws emerged from our survey, varying from the clinical indications for EEG recording and their prescribers, technical procedures of EEG execution, and personal protection of neurophysiology technologists. These were due to the lack of Italian recommendations for EEG recording during the phase 1 of the Covid-19 pandemic, which were then published by the most representative EEGrelated scientific societies (http://www.sinc-italia.it; http:// www.lice.it; http://www.aitn.it).

\section{Compliance with ethical standards}

Conflict of interest The authors declare that they have no conflicts of interest.

Research involving human participants and/or animals Not applicable.
Informed consent all the participants signed an informed consent before starting the questionnaire, the sign of informed consent was mandatory to fill the online questionnaire.

Ethical approval None.

\section{References}

1. (2020) Centers for Medicare and Medicaid Services. Non-emergent, elective medical services, and treatment recommendations. In: https://www.cms.gov/files/document/31820-cms-adultelective-surgery-and-procedures-Recomm

2. (2020) Interim guidance for healthcare facilities: preparing for community transmission of Covid-19 in the United States. In: Available at: https://www.cdc.gov/coronavirus/2019-ncov/hcp/guidance-hcf. html?CDC_AA_refVal=https $\% 3 \mathrm{~A} \% 2 \mathrm{~F} \% 2 \mathrm{Fwww} . \mathrm{cdc} . g o v \%$ 2Fcoronavirus $\% 2$ F2019-ncov $\% 2$ Fhealthcare-facilities $\%$ 2Fguidance-hcf.html

3. Stahel PF (2020) How to risk-stratify elective surgery during the Covid-19 pandemic? Patient Saf Surg 14:8. https://doi.org/10.1186/ s13037-020-00235-9

4. Klein BC, Busis NA (2020) Covid-19 is catalyzing the adoption of teleneurology. Neurology 94:903-904

5. Hurley D (2020) Neurologists scramble to respond to Covid-19 with telemedicine: the challenges and opportunities. Neurol Today 20:18-19

6. Assenza G, Lanzone J, Brigo F et al (2020) Epilepsy care at the time of Covid-19 pandemic in Italy: risk factors for seizure worsening. Front Neurol

7. Assenza G, Mecarelli O, Tombini M, Pulitano P, Pellegrino G, Benvenga A, Assenza F, Campana C, di Pino G, di Lazzaro V (2015) Hyperventilation induces sympathetic overactivation in mesial temporal epilepsy. Epilepsy Res 110:221-227. https://doi.org/ 10.1016/j.eplepsyres.2014.12.003

8. Wasserman AJ, Patterson JL (1961) The cerebral vascular response to reduction in arterial carbon dioxide tension. J Clin Invest 40 : $1297-1303$

9. Society ACN (2020) Covid-19 Resources. https://www.acns.org/ practice/covid-19-resources

10. Grippo A, Assenza G, Scarpino M et al (2020) Electroencephalography during SARS-CoV-2 outbreak: practical recommendations from the Task Force of the Italian Society of Neurophysiology (SINC), the Italian League Against Epilepsy (LICE), and the Italian Association of Neurophysiology Technologists (AITN). Neurol Sci

11. della Salute M (2020) Ministero della Salute Italiano - Circolare Ministeriale n. 7422 del 16.03.2020: Linee di indirizzo per la rimodulazione dell'attività programmata differibile in corso di emergenza da Covid-19. http://www.trovanorme.salute.gov.it/ norme $/$ renderNormsanPdf?anno $=2020 \&$ codLeg $=73675 \&$ parte $=$ $1 \&$ serie $=$ null

12. Sinha SR, Sullivan LR, Sabau D, Orta DSJ, Dombrowski KE, Halford JJ, Hani AJ, Drislane FW, Stecker MM (2016) American clinical neurophysiology society guideline 1 : minimum technical requirements for performing clinical electroencephalography. Neurodiagn J 56:235-244

Publisher's note Springer Nature remains neutral with regard to jurisdictional claims in published maps and institutional affiliations. 